\title{
Aspectos médicos del tratamiento quirúrgico de la obesidad
}

\author{
Medical issues of surgical treatment of obesity
}

\author{
María José Morales ${ }^{\mathrm{a}}$, María Jesús Díaz-Fernández ${ }^{\mathrm{b}}$, Assumpta Caixàs ${ }^{\mathrm{c}}$, Fernando \\ Cordido ${ }^{\mathrm{d}}$, Grupo de Trabajo sobre Obesidad de la Sociedad Española de Endocrinología \\ y Nutrición \\ ${ }^{a}$ Servicio de Endocrinología y Nutrición, Hospital Meixoeiro, Complexo Hospitalario Universitario de Vigo, Vigo, \\ Pontevedra, España \\ ${ }^{b}$ Servicio de Endocrinología y Nutrición, Hospital Valle del Nalón, Langreo, Asturias, España \\ ${ }^{c}$ Servicio de Endocrinología y Nutrición. Hospital de Sabadell, Corporació Sanitària e Institut Universitari Parc \\ Taulí, Universitat Autònoma de Barcelona, Sabadell, Barcelona, España \\ d Servicio de Endocrinología, Complexo Hospitalario Universitario A Coruña, Departamento de Medicina, \\ Universidad de A Coruña, A Coruña, España
}

\section{Introducción}

El fracaso del tratamiento médico en la mayoría de los pacientes con obesidad mórbida (OM) hace necesaria la cirugía bariátrica (CB) para conseguir una pérdida de peso efectiva y mantenida en el tiempo ${ }^{1}$. Sin embargo, tanto la situación clínica de estos pacientes como la complejidad de la cirugía limitan su indicación al cumplimiento de una serie de criterios de inclusión (tabla 1), requiriéndose una cuidadosa valoración preoperatoria y un estrecho seguimiento postoperatorio por parte de un equipo hospitalario multidisciplinar que trabaje de forma coordinada y protocolizada ${ }^{2}$ and 3 . Dentro del equipo, las funciones del médico clínico deben centrarse en la selección y preparación preoperatoria de los pacientes y en el seguimiento médico y nutricional postoperatorio a corto y largo plazo.

Tabla 1. Indicaciones de la cirugía bariátrica

\author{
Obesidad con $\mathrm{IMC} \geq 40 \mathrm{Kg} / \mathrm{m} 2 \mathrm{o}>35 \mathrm{Kg} / \mathrm{m} 2$ con comorbilidades mayores \\ Edad entre 18 y 65 años (flexible, según caso clínico) \\ Duración de la obesidad mórbida superior a 5 años \\ Fracasos previos del tratamiento médico \\ Ausencia de enfermedad psiquiátrica y/o trastornos de la conducta alimentaria activos y de abuso de drogas y/o alcohol \\ Capacidad para comprender, comprometerse y adherirse a los cambios que supone la cirugía \\ Ausencia de enfermedad endocrinológica que justifique la obesidad y de otro trastorno que contraindique la cirugía
}

IMC: índice de masa corporal. 
El aumento progresivo del número de pacientes candidatos a $\mathrm{CB}$ exige que cada vez más especialistas deban familiarizarse con su manejo, disponiendo de información actualizada y de buena calidad. Este artículo pretende responder a las cuestiones médicas que con frecuencia se plantean en el abordaje clínico de estos pacientes.

\section{Justificación del tratamiento quirúrgico de la obesidad}

La CB es el tratamiento más eficaz para los adultos con OM, como demuestran los estudios que comparan diferentes procedimientos quirúrgicos con intervenciones no quirúrgicas, no sólo en pérdida de peso, sino también en resolución de las comorbilidades asociadas a la obesidad ${ }^{4,5,6,7}$ and 8 y en reducción de la mortalidad ${ }^{9}$ and 10 . Existe debate sobre su indicación en la adolescencia, pero podría considerarse en casos graves bien seleccionados ${ }^{11}$. Recientemente, la Asociación Americana de Diabetes ha incluido por primera vez la $\mathrm{CB}$ en los algoritmos de manejo de la diabetes mellitus tipo 2 (DM-2) ${ }^{12}$ ante las evidencias

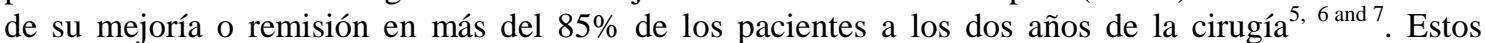
resultados varían según el procedimiento quirúrgico, siendo mejores en aquellos que, además de la reducción gástrica, incluyen una derivación parcial del intestino delgado ${ }^{13}$.

\section{Estudios a realizar antes de la cirugía}

La valoración preoperatoria debe incluir una historia clínica con datos sobre la evolución ponderal, hábitos tóxicos, patrón de alimentación, actividad física, enfermedades psiquiátricas y antecedentes de procesos por aparatos, especificando los tratamientos previos y la medicación actual. La anamnesis y las exploraciones complementarias valorarán, además, enfermedades asociadas a la obesidad o causas de obesidad secundaria ${ }^{3}$ (tabla 2 ).

Se debe informar al paciente de las opciones quirúrgicas (incluyendo los resultados reales y las posibles complicaciones) y solicitar un informe psiquiátrico para descartar trastornos del comportamiento alimentario u otra patología que contraindique la cirugía y para asegurar la idoneidad del paciente ${ }^{14}$. Si se considera apto por Psiquiatría, se remitirá al Servicio de Cirugía que, en base a la situación del paciente y a su propia experiencia, seleccionará finalmente la técnica quirúrgica ${ }^{15}$. El control médico y nutricional prequirúrgico para optimizar el tratamiento de las comorbilidades y mejorar los hábitos alimentarios, intentando una pérdida moderada de peso, se realizará con el apoyo de dietistas o diplomados en nutrición.

\section{Técnicas quirúrgicas más utilizadas}

Los procedimientos quirúrgicos pueden dividirse en tres grupos ${ }^{15}$ and 16 :

A. Restrictivos (limitan la capacidad gástrica).

- Gastroplastia vertical en banda (GVB).

- Banda gástrica ajustable (BGA).

- Gastrectomía tubular (GT).

B. Malabsortivos (derivan parte del intestino delgado, dificultando la digestión y absorción de los alimentos). Abandonados por sus graves complicaciones.

C. Mixtos (incluyen ambos componentes).

- Bypass gástrico en Y de Roux (BGYR), preferentemente restrictivo.

- Derivación biliopancreática y sus variantes, como el cruce duodenal (DBP/CD), preferentemente malabsortivos. 
Actualmente, el abordaje de elección es la laparoscopia, al haber demostrado una menor estancia hospitalaria, una recuperación más rápida y una menor incidencia de complicaciones respecto a la cirugía abierta, con resultados semejantes en pérdida de peso, calidad de vida y resolución de las comorbilidades. El BGYR y la BGA son los procedimientos laparoscópicos más utilizados y con una mejor evidencia científica en relación riesgo-beneficio. La GT se considera una técnica emergente ${ }^{8 \text { and } 15}$.

Tabla 2. Valoración médica preoperatoria

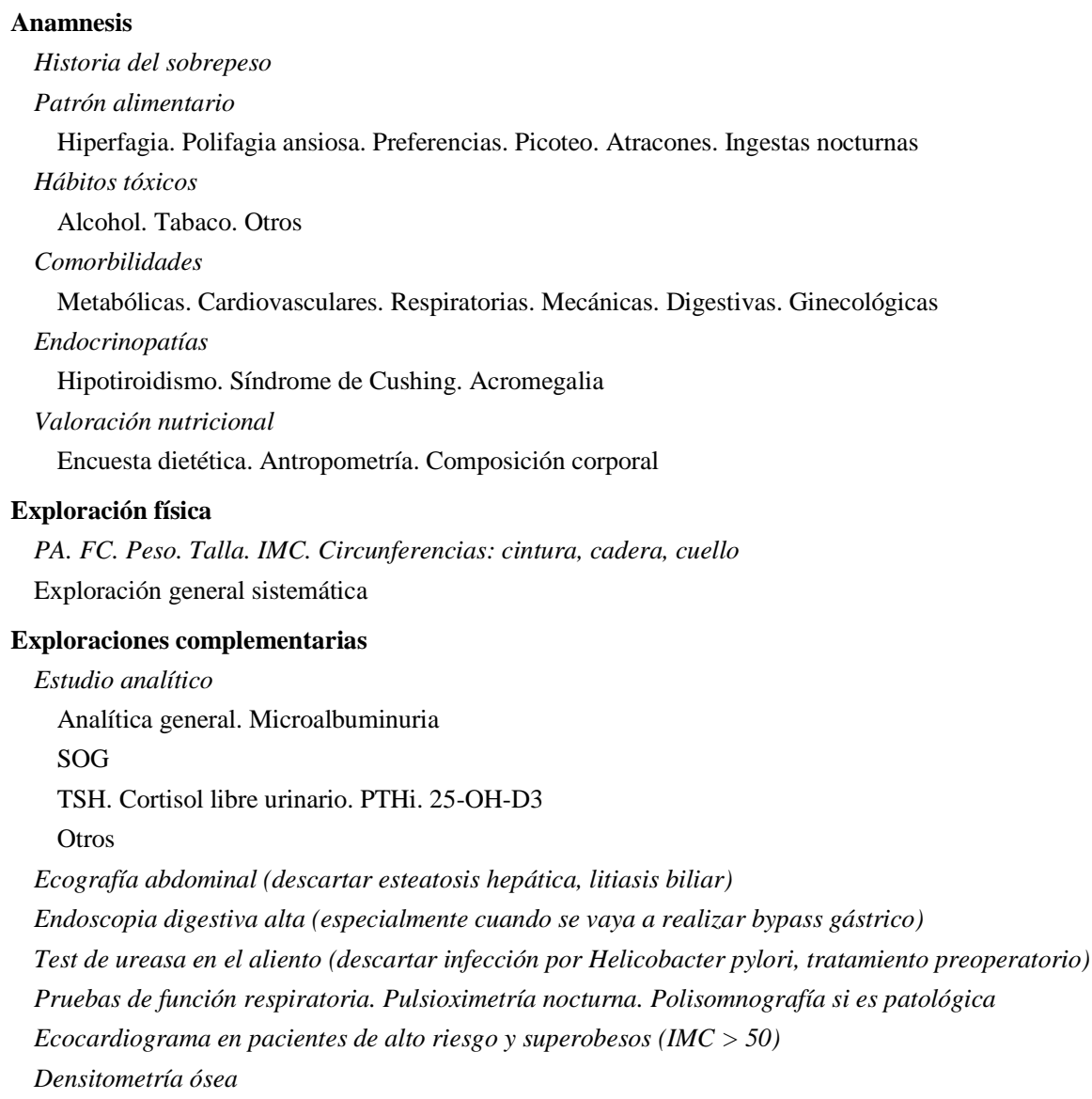

FC: frecuencia cardiaca; IMC: índice de masa corporal; PA: presión arterial; PTHi: paratirina intacta; SOG: sobrecarga oral de glucosa (75 gramos); TSH: tirotropina; $25-\mathrm{OH}-\mathrm{D}_{3}$ : calcifediol.

\section{Optimización de la preparación preoperatoria}

La pérdida de peso en el preoperatorio inmediato reduce el tamaño hepático y la grasa perivisceral, facilitando el acceso quirúrgico y reduciendo la duración de la intervención. Los métodos más utilizados incluyen las dietas comerciales de muy bajo contenido calórico ${ }^{17}$ y el balón intragástrico ${ }^{18}$.

La presencia de comorbilidades (DM, hipertesión arterial [HTA], trastornos respiratorios) hace necesario su control antes de la cirugía ${ }^{2}$ and 3 . Además, en la OM son frecuentes las deficiencias de micronutrientes (vitamina $\mathrm{D}$, otros), requiriéndose su valoración sistemática y la corrección preoperatoria de los déficits presentes ${ }^{19}$. 


\section{Planificación del seguimiento postoperatorio}

La CB conlleva riesgo de morbimortalidad por complicaciones técnicas, nutricionales e incluso psiquiátricas, siendo imprescindible un seguimiento multidisciplinar a corto y largo plazo. En la tabla 3 se muestra la programación de las revisiones postoperatorias.

A efectos prácticos, el seguimiento médico puede dividirse en tres etapas ${ }^{20}$ :

1. Periodo perioperatorio (hospitalización y primer mes postoperatorio)

2. Periodo postoperatorio temprano (pérdida de peso, del segundo mes a los 12-18 meses).

3. Periodo postoperatorio tardío (tras estabilización del peso, indefinido).

\section{Periodo perioperatorio}

El seguimiento estará centrado en la planificación del tratamiento nutricional. La progresión de la alimentación debe ser gradual, desde una dieta líquida muy fraccionada a una dieta sólida en 1-3 meses, según la tolerancia digestiva y el tipo de cirugía (con un periodo de transición más prolongado cuanto mayor sea el componente restrictivo $)^{21}$. Ante la dificultad para cubrir los requerimientos proteicos (1-1,5 $\mathrm{g} / \mathrm{Kg} / \mathrm{día})^{22}$, se aconseja asociar un suplemento hiperproteico o un módulo proteico que aporte unos 20-30 gramos adicionales al día, hasta finalizar el periodo de transición ${ }^{23}$ (tabla 4). Todos los pacientes requieren suplementación de multivitaminas y minerales de forma indefinida para prevenir deficiencias de micronutrientes (dosis variable, según técnica quirúrgica). Inicialmente son preferibles los preparados líquidos o masticables para evitar impactaciones. Se deben administrar suplementos orales de hierro $(\mathrm{Fe})$ en mujeres menstruantes y suplementos de calcio con vitamina D tras las técnicas derivativas. En algunos casos serán necesarios otros suplementos posteriormente ${ }^{2,3}$ and 20 . 
Tabla 3. Componentes y calendario del seguimiento médico postoperatorio. Individualizar según técnica, evolución clínica y complicaciones

\begin{tabular}{|c|c|c|c|c|c|c|c|c|}
\hline & $1 \mathrm{~m}$ & $3 \mathrm{~m}$ & $6 \mathrm{~m}$ & $12 \mathrm{~m}$ & $18 \mathrm{~m}$ & $24 \mathrm{~m}$ & Anual & 5 años \\
\hline 1. Antropometría & $\mathrm{x}$ & $\mathrm{x}$ & $\mathrm{x}$ & $\mathrm{x}$ & $\mathrm{x}$ & $\mathrm{x}$ & $\mathrm{x}$ & \\
\hline 2. Tolerancia digestiva & $\mathrm{x}$ & $\mathrm{x}$ & $\mathrm{x}$ & $\mathrm{x}$ & $\mathrm{x}$ & $\mathrm{x}$ & $\mathrm{x}$ & \\
\hline 3. Cumplimiento & $\mathrm{x}$ & $\mathrm{x}$ & $\mathrm{x}$ & $\mathrm{x}$ & $\mathrm{x}$ & $\mathrm{x}$ & $\mathrm{x}$ & \\
\hline 4. Comorbilidades & $\mathrm{x}$ & $\mathrm{x}$ & $\mathrm{x}$ & $\mathrm{x}$ & $\mathrm{x}$ & $\mathrm{x}$ & $\mathrm{x}$ & \\
\hline 5. Analítica* & $\mathrm{x}$ & $\mathrm{x}$ & $\mathrm{x}$ & $\mathrm{x}$ & $\mathrm{x}$ & $\mathrm{x}$ & $\mathrm{x}$ & \\
\hline 25-OH-D3 & & $\mathrm{x}$ & $\mathrm{x}$ & $\mathrm{x}$ & $\mathrm{x}$ & $\mathrm{x}$ & $\mathrm{x}$ & \\
\hline PTHi & & & $\mathrm{x}$ & $\mathrm{x}$ & $\mathrm{x}$ & $\mathrm{x}$ & $\mathrm{x}$ & \\
\hline 6. Complicaciones & $\mathrm{x}$ & $\mathrm{x}$ & $\mathrm{x}$ & $\mathrm{x}$ & $\mathrm{x}$ & $\mathrm{x}$ & $\mathrm{x}$ & \\
\hline 7. DMO & & & & $\mathrm{x}$ & --- & $\mathrm{x}$ & $\mathrm{x}^{* *}$ & \\
\hline 8. Calidad de vida & & & & $\mathrm{x}$ & & $\mathrm{x}$ & $\mathrm{x}$ & \\
\hline 9. Resultados globales & & & & & & & & $\mathrm{x}$ \\
\hline \multicolumn{9}{|l|}{ 1. Antropometría } \\
\hline \multicolumn{9}{|l|}{ Peso actual. Peso perdido. \% PSP } \\
\hline \multicolumn{9}{|l|}{ IMC actual. IMC perdido. \% IMCP } \\
\hline \multicolumn{9}{|c|}{ Evolución de circunferencia de cintura, índice cintura/cadera } \\
\hline \multicolumn{9}{|l|}{ 2. Tolerancia digestiva } \\
\hline \multicolumn{9}{|l|}{ Vómitos, dolor abdominal, diarrea } \\
\hline \multicolumn{9}{|c|}{ Adaptación a la dieta, hábitos alimentarios } \\
\hline \multicolumn{9}{|l|}{ 3. Cumplimiento del tratamiento } \\
\hline \multicolumn{9}{|l|}{ Plan de alimentación } \\
\hline \multicolumn{9}{|l|}{ Actividad física } \\
\hline \multicolumn{9}{|l|}{ Toma de suplementos } \\
\hline \multicolumn{9}{|c|}{ 4. Evolución de comorbilidades preoperatorias } \\
\hline \multicolumn{9}{|c|}{ Diabetes mellitus. HTA. Dislipidemia. SAOS. Otras } \\
\hline \multicolumn{9}{|l|}{ 5. Analítica* } \\
\hline \multicolumn{9}{|c|}{$\begin{array}{l}\text { Hemograma completo, coagulación, bioquímica con perfil hepático, ferritina, ácido fólico, vitamina B12, calcio, fósforo, } \\
\text { albúmina, transferrina, calciuria. Otros micronutrientes en caso necesario }\end{array}$} \\
\hline \multicolumn{9}{|l|}{ 6. Complicaciones postoperatorias } \\
\hline \multicolumn{9}{|l|}{ Técnicas. Nutricionales. Psiquiátricas } \\
\hline \multicolumn{9}{|l|}{ 7. DMO } \\
\hline \multicolumn{9}{|c|}{ **Si DMO baja o en descenso a los $24 \mathrm{~m}$, medir DMO anualmente } \\
\hline \multicolumn{9}{|l|}{ 8. Calidad de vida relacionada con la salud } \\
\hline \multicolumn{9}{|l|}{ Test genérico y/o específico } \\
\hline \multicolumn{9}{|l|}{ 9. Resultados globales } \\
\hline Eficacia+seguridad+calidad de vida & & & & & & & & \\
\hline
\end{tabular}

DMO: densidad mineral ósea; HTA: hipertensión arterial; IMC: índice de masa corporal; IMCP: IMC perdido; PSP: sobrepeso perdido; SAOS: síndrome apnea obstructiva del sueño. 
Tabla 4. Planificación de la alimentación y de los suplementos en el periodo perioperatorio

\section{Objetivos}

Facilitar tolerancia digestiva

Reeducación nutricional: adaptación a los cambios anatómicos y funcionales inducidos por la cirugía

\section{Características de la dieta}

Inicialmente líquida y pobre en residuos

Repartida en 6 tomas. Pequeños volúmenes en cada toma (50 ml)

Progresiva en cantidad y consistencia

\section{Normas generales}

Tránsito gastrointestinal alto antes de iniciar alimentación oral

Primera semana. Dieta líquida

Día 1: probar tolerancia

Días 2-3 ( ${ }^{\text {a }}$ fase): líquidos claros (50ml cada $15^{\prime}$ ')+suplemento proteico (20-30 g/día)*

Días 4-7 ( $2^{\mathrm{a}}$ fase): igual+líquidos espesos+suplemento proteico (20-30 g/día)*

Segunda a quinta semanas. Dieta semilíquida (tipo puré)

Igual+puré con leche, carne blanca, pescado blanco o huevos triturados+suplemento proteico (20 g/día)*

A partir del segundo mes. Dieta triturada/blanda

Suficiente en proteínas (> 60 g/día), baja en grasa y sin adición de azúcares simples

Como alternativa (comidas fuera de casa), pueden consumirse productos comerciales de textura modificada para adultos $* *$

\section{*Fórmulas hiperproteicas hipocalóricas o normocalóricas. Gramos de proteínas (g)}

OPTISOURCE® $(7,5 \mathrm{~g} / 100 \mathrm{ml})$

OPTISOURCE®PLUS $(7 \mathrm{~g} / 100 \mathrm{ml})$

VegeFast Diet $\left.{ }^{(7,4} \mathrm{g} / 100 \mathrm{ml}\right)$

VegeStart Complet ${ }(7,8-8 \mathrm{~g} / 100 \mathrm{ml})$

\section{Módulo proteico}

PROTEÍNA-NM®, Vegenat-med Proteína®(10 g/sobre)

\section{**Alimentos de textura modificada para adultos}

RESOURCE®Puré (tarros) $(5 \mathrm{~g} / 100 \mathrm{~g})$

\section{Suplementos de micronutrientes}

Complejo de multivitaminas-minerales de forma indefinida

Suplementos de sulfato ferroso en mujeres con menstruaciones

Suplementos de calcio (+ vitamina D) en las técnicas con componente derivativo

Suplementos específicos (Vit. B1, Vit. B12, ácido fólico, Vit. D, Vit. A, Vit. K, magnesio, cinc, otros) en caso necesario 
Se caracteriza por una pérdida acelerada de peso y por la aparición de problemas de tolerancia digestiva (por ejemplo vómitos, síndrome de dumping), sobre todo durante los primeros meses.

Los vómitos iniciales son más frecuentes en las técnicas restrictivas, por la limitación de la ingesta, pero si persisten o se asocian a disfagia, deben descartarse complicaciones técnicas, como estenosis del estoma o de la anastomosis gastroyeyunal ${ }^{24}$, que puede resolverse mediante dilatación neumática con balón por endoscopia ${ }^{25}$. Estos pacientes tienen un riesgo elevado de deshidratación, deficiencias de micronutrientes (especialmente de tiamina y otras vitaminas del grupo B), malnutrición y complicaciones metabólicas como gota, litiasis renal o colelitiasis ${ }^{3 \text { and } 20}$, precisando una vigilancia nutricional intensiva y la administración profiláctica de tiamina por vía parenteral y posteriormente oral para prevenir la presentación de complicaciones neurológicas agudas graves, como la encefalopatía de Wernicke y la "neuropatía aguda poscirugía de reducción gástrica"26 (tabla 5). 
Tabla 5. Actitud en los pacientes operados con vómitos y/o diarrea

\section{Vómitos ocasionales}

Educación nutricional

Beber con frecuencia pequeñas cantidades $(50 \mathrm{ml})$ de agua o líquidos acalóricos fuera de las comidas

\section{Vómitos persistentes}

Valoración clínica y analítica urgente. Descartar deshidratación, hipopotasemia y/o hipomagnesemia

Deshidratación leve: bebidas deportivas de reposición diluidas al 50\%

Empeoramiento o deshidratación grave: hospitalización para rehidratación y suplementación IV

Administración profiláctica de suplementos de tiamina por vía parenteral (100mg/día durante 7-14 días, según evolución).

Mantener posteriormente suplementación oral hasta la recuperación completa

Solicitar endoscopia digestiva alta para descartar estenosis del estoma o de la anastomosis y/o úlceras marginales

Si estenosis, dilatación neumática con balón en el momento del diagnóstico

Dilataciones repetidas hasta capacidad para tolerar comidas sólidas

En pacientes sintomáticos o con úlceras marginales, descartar infección por Helicobacter pylori

Descartar trastorno del comportamiento alimentario

\section{Diarrea persistente}

Modificaciones dietéticas: dieta pobre en grasa. Dieta sin lactosa

Descartar sobrecrecimiento bacteriano

Cultivo de las secreciones intestinales si es posible

Test de hidrógeno en el aliento con lactulosa (sensibilidad limitada)

Antibioterapia empírica con metronidazol ante la sospecha clínica

Descartar esteatorrea

Dietas pobres en grasa enriquecidas con triglicéridos de cadena media

Algunos autores recomiendan enzimas pancreáticas

Descartar enfermedad celíaca

Dieta sin gluten

\section{Vigilar el desarrollo de malnutrición proteica}

En los pacientes con vómitos persistentes o ingesta muy restrictiva

En los pacientes intervenidos con técnicas de predominio malabsortivo, especialmente si diarrea

Ante cualquier circunstancia que aumente los requerimientos o produzca una mala utilización de las proteínas (infección, cirugía, ciertos tratamientos como corticoides o quimioterápicos, embarazo, etc.)

Monitorizar los valores séricos de albúmina y transferrina

Malnutrición leve (albúmina 2,8-3,5 g/100 ml; transferrina 150-200 mg/100 ml)

Modificaciones dietéticas+fórmula hiperproteica o suplemento proteico oral

Malnutrición moderada-grave (albúmina $<2,8 \mathrm{~g} / 100 \mathrm{ml}$; transferrina $<150 \mathrm{mg} / 100 \mathrm{ml}$ )

Hospitalización para soporte nutricional (generalmente parenteral) durante 3-4 semanas

Precaución al iniciar NPT para evitar síndrome de realimentación (edema, hipokaliemia, hipofosfatemia, hipomagnesemia)

Valorar la necesidad de cirugía de reconversión bariátrica en los pacientes dependientes de NPT

NPT: nutrición parenteral total. 
El síndrome de dumping aparece en los procedimientos derivativos por el rápido vaciamiento del reservorio gástrico. Su frecuencia varía, pudiendo ocurrir inicialmente hasta en el $75 \%$ de los casos después del BGYR ${ }^{27}$. La llegada de alimentos hiperosmolares no digeridos (por ejemplo bebidas azucaradas, helados, pasteles) al intestino delgado produce distensión intestinal y secreción de péptidos intestinales, originando entrada de líquido en la luz, hipovolemia, vagotonía e hiperinsulinismo, responsables de la sintomatología. Según algunos autores, esto podría favorecer la anorexia postoperatoria y la aversión por los dulces que sufren muchos de los pacientes sometidos a BGYR y ser responsable, al menos en parte, de su mayor eficacia con respecto a las técnicas restrictivas ${ }^{27}$. El llamado dumping tardío, asociado a hipoglucemia reactiva, suele manifestarse en los primeros meses, pero se han descrito casos de hipoglucemia grave por hiperinsulinismo asociado a nesidioblastosis que precisaron pancreatectomía, hasta 9 años después del BGYR ${ }^{28}$. El manejo inicial se basa en modificaciones dietéticas: disminuir el volumen y aumentar la frecuencia de las tomas, comenzar la comida por el alimento proteico, evitar los líquidos durante las comidas e incrementar la ingesta de fibra soluble ${ }^{23}$. Se ha recomendado la administración de acarbosa y, en los casos graves, de análogos de la somatostatina antes de recurrir a la cirugía ${ }^{3}$.

La formación de cálculos biliares, relacionada con la pérdida rápida de peso, puede dar síntomas hasta en el $40 \%$ de los casos, por lo que algunos cirujanos recomiendan una colecistectomía profiláctica. El tratamiento médico con un agente solubilizador (ácido ursodeoxicólico) durante 6 meses, si se tolera, es eficaz como profilaxis ${ }^{29}$.

El riesgo de litiasis renal por oxalato cálcico aumenta con los procedimientos derivativos, sobre todo si hay deshidratación. Además de incentivar la ingesta hídrica, se debe indicar una dieta baja en oxalatos con suplementación adecuada de calcio y de citrato potásico. En los pacientes con antecedentes de hiperuricemia sintomática (gota, litiasis renal) se recomienda tratamiento profiláctico desde antes de la intervención ${ }^{3}$.

La pérdida de cabello es frecuente en los primeros meses, habiéndose relacionado con deficiencia de proteínas y de cinc. Suele recuperarse sin tratamiento ${ }^{20}$.

\section{Periodo postoperatorio tardio}

Una vez estabilizado el peso corporal, es necesario mantener el seguimiento a largo plazo para detectar y tratar a tiempo las complicaciones tardías, especialmente las deficiencias nutricionales. Además de los riesgos nutricionales intrínsecos del procedimiento quirúrgico (mayores con los malabsortivos), la calidad de la alimentación y el grado de cumplimiento terapéutico condicionarán la aparición de complicaciones $^{30}$.

Las técnicas restrictivas, al no modificar la fisiología digestiva, presentan menor riesgo nutricional a largo plazo, pero la limitación de volumen obliga a tomas muy fraccionadas de comida triturada, por lo que pueden aparecer vómitos y alteraciones de la conducta alimentaria, con el consiguiente fracaso de la intervención ${ }^{15,27,31}$.

El BGYR y la DBP/CD comportan mayor riesgo de anemia y de otras complicaciones nutricionales al evitar el paso de los alimentos por gran parte del estómago, la totalidad del duodeno y una porción variable del intestino delgado, disminuyendo la absorción de Fe, vitamina B12, ácido fólico, calcio y otros micronutrientes ${ }^{3,20,31,32}$

Las deficiencias de Fe y de $\mathrm{B}_{12}$ son las más comunes. La disminución de la secreción gástrica de ácido clorhídrico, el déficit de factor intrínseco y la intolerancia a alimentos ricos en estos nutrientes (carnes rojas), favorecen su aparición. La anemia ferropénica afecta especialmente a mujeres con menstruaciones sometidas a BGYR y DBP (hasta un 50\%), pudiendo aparecer en los primeros meses e incluso años después de la intervención. El riesgo de deficiencia de $\mathrm{B}_{12}$ aumenta progresivamente a partir del primer año, al ser el depósito corporal muy superior a las necesidades diarias. Su frecuencia a largo plazo podría llegar al 70\%, sobre todo después del BGYR, si no se suplementa ${ }^{33,34}$. 
Las técnicas de predominio malabsortivo (DBP/CD, BG distal) producen deficiencia de vitamina $\mathrm{D}$ y de otras vitaminas liposolubles con mayor frecuencia que el BGYR, debido a la esteatorrea resultante de la mezcla retrasada y defectuosa del bolo alimenticio con las secreciones bilio-pancreáticas en el tramo final del intestino delgado (canal común) ${ }^{31,35}$. La aparición de enfermedad ósea metabólica, superior al $60 \%$, se asocia principalmente a disminución de la ingesta de calcio (intolerancia a lácteos), a malabsorción de calcio y vitamina D con hiperparatiroidismo secundario y a la presencia de malnutrición proteica, presentando mayor riego los pacientes con un canal común más corto $(<100 \mathrm{~cm})$. Los valores séricos de calcio, fósforo y fosfatasa alcalina son a menudo normales y no son útiles para el despistaje. Es obligado monitorizar la concentración de paratirina intacta (PTHi) y de 25-OH-D3, ajustando los suplementos hasta normalizar sus valores, así como evaluar periódicamente la densidad mineral ósea y la necesidad de tratamiento antiosteoporótico ${ }^{36,37}$.

La deficiencia subclínica de vitamina A podría afectar a largo plazo a más del $60 \%$ de los casos de DBP según algunos estudios ${ }^{35}$, pero los problemas oftalmológicos son poco frecuentes ( $3 \%$ de ceguera nocturna en la serie de Scopinaro $^{38}$ ). Los suplementos profilácticos no evitan la deficiencia en todos los casos, por lo que está indicada su monitorización. Las deficiencias de otras vitaminas liposolubles no suelen tener repercusión clínica.

Los pacientes con diarrea crónica presentan riesgo de deficiencia de cinc y de magnesio. Las deficiencias de cobre y de selenio, generalmente subclínicas, deben descartarse en caso de anemia persistente con valores normales de $\mathrm{Fe}$, folato y $\mathrm{B} 1^{23,33}$.

En la tabla 6 se muestran las recomendaciones para la prevención y el tratamiento de las deficiencias de micronutrientes.

Tabla 6. Prevención y tratamiento de las deficiencias de micronutrientes

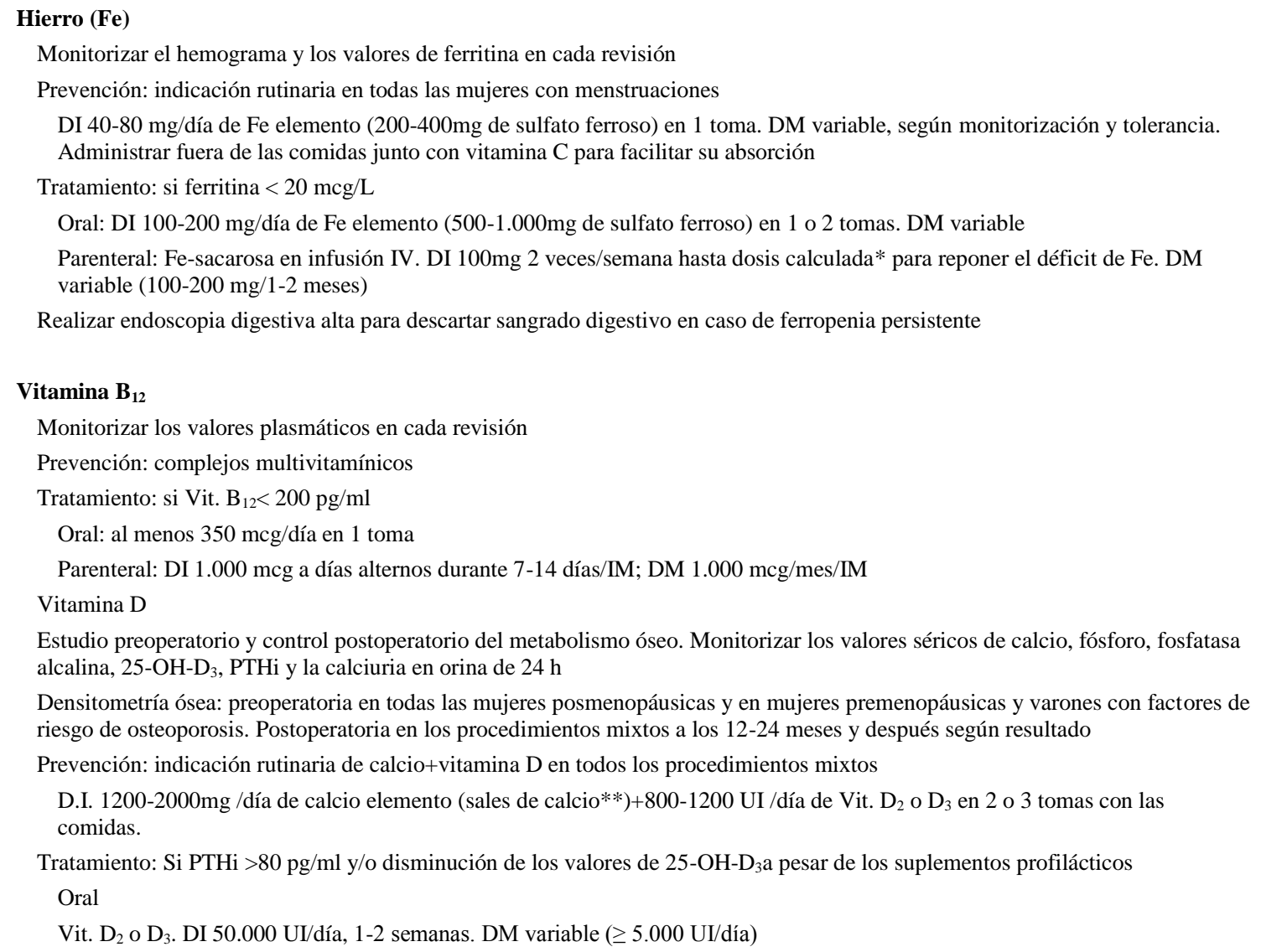


25-OH-D 3 . DI 180.000 UI/semana, 2-4 semanas. DM variable (16.000-32.000 UI/semana)

Parenteral: hipocalcemia sintomática, malabsorción grave

1,25- $(\mathrm{OH})_{2}-\mathrm{D}_{3}$ (calcitriol). DI 1-2 mcg/48 h/IV hasta normalización de la calcemia. DM variable (1-2 mcg/semana/IV)

\section{Otras vitaminas liposolubles}

Monitorizar anualmente los valores de vitaminas A y E y la actividad de protrombina/INR (o la Vit. $\mathrm{K}_{1}$ ) en los procedimientos de predomino malabsortivo y si diarrea persistente

Prevención: complejos multivitamínicos

Tratamiento: si deficiencia, según valores de referencia

Vitamina A. Oral: DI 50.000 UI/día durante 1 semana. DM 50.000 UI 2 veces/semana o 5.000-10.000 UI/día con vigilancia de las concentraciones plasmáticas por riesgo de hepatotoxicidad

Vitamina K (si valores de INR > 1,4). Oral: $1 \mathrm{mg} /$ día

Vitamina E. Oral: 400 UI/día

\section{Vitamina $\mathbf{B}_{1}$ (tiamina)}

No monitorizar rutinariamente los niveles séricos

Prevención: en pacientes de riesgo por vómitos o ingesta muy restrictiva

Oral: $10 \mathrm{mg} /$ día. Parenteral: $100 \mathrm{mg} /$ día/IM si intolerancia digestiva. Mantener suplementación oral hasta la resolución de los síntomas

Tratamiento: si síntomas neurológicos, antes de administrar soluciones de dextrosa

Parenteral: DI 100 mg/día durante 7-14 días/IM o IV lenta. Mantener suplementación oral hasta la máxima recuperación neurológica

\section{Ácido fólico}

Monitorizar periódicamente los niveles séricos

Prevención: complejos multivitamínicos que aporten $400 \mathrm{mcg} /$ día

Tratamiento: oral: 10-20 mg/día en 2 tomas antes de las comidas

Cinc, magnesio, cobre, selenio

Monitorizar los niveles de cinc y de magnesio si diarrea persistente. No administrar suplementos de cinc sin documentar su deficiencia: puede inducir deficiencia de cobre y anemia secundaria

Monitorizar los niveles de cobre y de selenio en pacientes con anemia resistente al tratamiento con Fe, ácido fólico y vitamina B12

Prevención: complejos multivitamínicos

Tratamiento: si deficiencia

Cinc. Oral: 40-100 mg/día (176-440mg de sulfato de cinc) en 1 o 2 tomas, con las comidas

Magnesio. Oral: 350-500 mg/día (3-5g de lactato de magnesio) en 3 o 4 tomas con las comidas. Precaución por posible empeoramiento de la diarrea. Parenteral: si deficiencia grave, con síntomas neuromusculares o cardiacos

Cobre. Oral: $1,5-3 \mathrm{mg} /$ día

Selenio. Oral: 75-100 mcg/día

DI: dosis inicial; DM: dosis de mantenimiento; Fe: hierro; IM: intramuscular; INR: ratio normalizada internacional; IV: intravenosa; PTHi: paratirina intacta.

* Para el cálculo de la dosis se puede utilizar la fórmula: déficit de $\mathrm{Fe}(\mathrm{mg})=$ peso $(\mathrm{Kg})$ x $(\mathrm{Hb}$ deseada - $\mathrm{Hb}$ actual [gr/L]) x 0,24 + 500 mg. La administración puede realizarse en el Hospital de Día.

** El citrato cálcico es la sal idónea pero hasta hace poco tiempo no existían preparados comercializados en nuestro país, por lo que suele utilizarse inicialmente carbonato cálcico, recurriendo a otras sales en caso de intolerancia.

La malnutrición proteica constituye la complicación potencial más grave de la $\mathrm{CB}$, pero actualmente es poco frecuente, incluso tras la $\mathrm{DBP}^{38}$. En la práctica suele ser secundaria a complicaciones técnicas o al incumplimiento de las recomendaciones nutricionales, afectando sobre todo a pacientes con vómitos y/o diarrea persistentes ${ }^{30,31}$. Su prevalencia oscila entre el $0-30 \%$, dependiendo del tamaño del reservorio gástrico, del diámetro del estoma gastroyeyunal, de las longitudes del canal alimentario (menor de 200 $\mathrm{cm}$ ) y del canal común (menor de $100 \mathrm{~cm}$ ) y de la presencia de sobrecrecimiento bacteriano ${ }^{38,39}$. Para prevenir su aparición, se debe valorar regularmente la ingesta nutricional, administrando un suplemento proteico si la ingesta proteica es inferior a 60 gramos/día. Los casos graves requieren hospitalización para soporte nutricional, generalmente parenteral ${ }^{3,40,41}$ (tabla 5). 
La figura 1 contiene un algoritmo actualizado para el manejo de las complicaciones nutricionales $\operatorname{postoperatorias~}^{42}$.

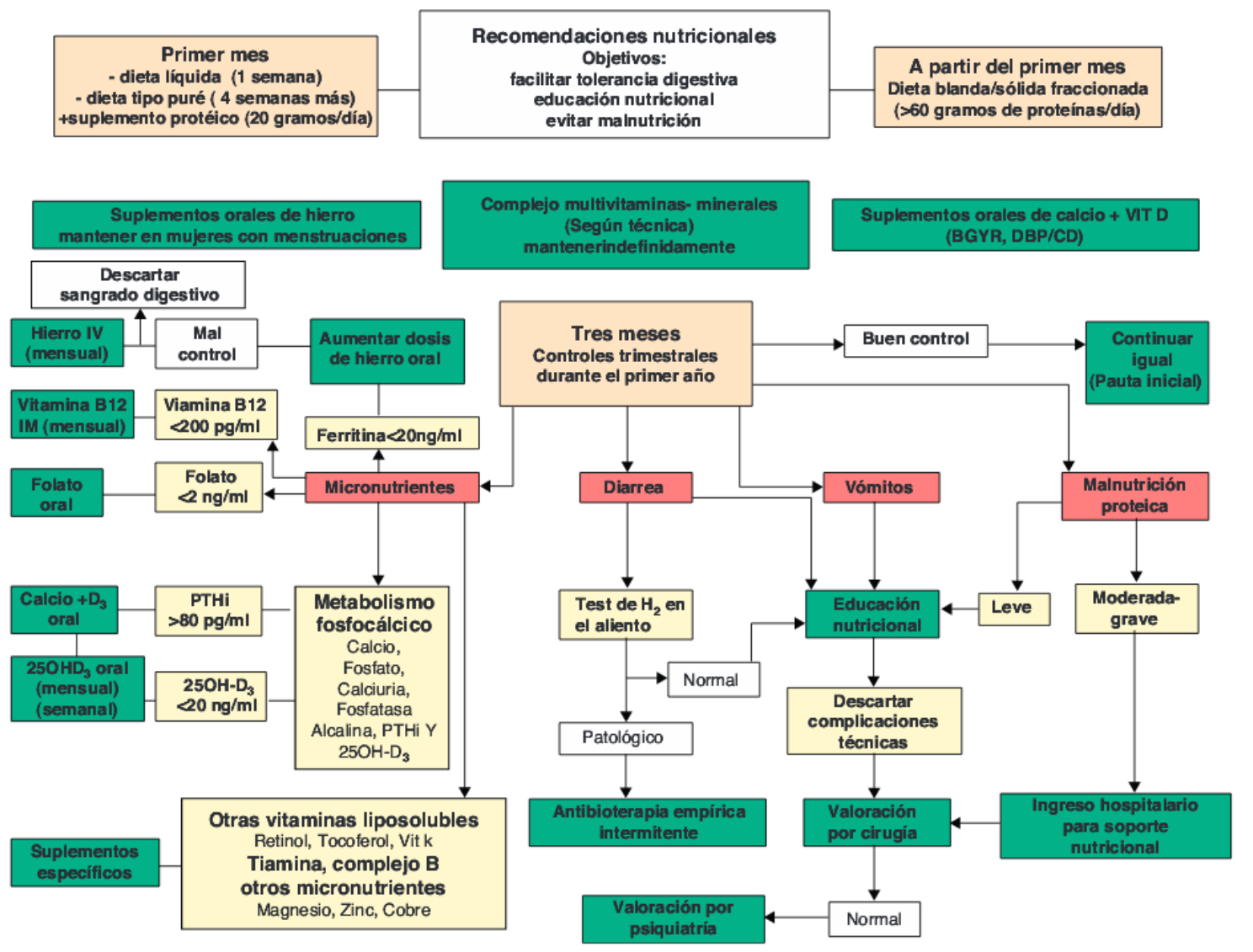

Figura 1. Cirugía bariátrica. Algoritmo de seguimiento postoperatorio.

\section{¿Qué precauciones se deben adoptar con respecto a la administración de fármacos en el} postoperatorio?

La mejoría de las comorbilidades preoperatorias inducida por la CB y la disminución sustancial de los requerimientos farmacológicos en el postoperatorio, obligan a frecuentes ajustes en el tratamiento de algunas de ellas, como la DM y la HTA, para evitar yatrogenia ${ }^{3,5}$ and 6.

En los pacientes con DM-2 se recomienda vigilar estrechamente la administración de insulina durante el periodo perioperatorio (valorando su indicación antes del alta) y evitar las sulfonilureas en el postoperatorio, por el riesgo de hipoglucemia. Sin embargo, es importante recordar que un pequeño porcentaje de los pacientes con OM presenta DM-1 y precisa insulina para su supervivencia (y para prevenir la cetoacidosis), por lo que su uso es obligado, incluso en ayunas.

La mayoría de los pacientes con tratamiento antihipertensivo antes de la CB seguirán necesitándolo en el momento del alta. Deben evitarse los diuréticos en los primeros meses por el riesgo de deshidratación y de trastornos hidroelectrolíticos, pudiendo reintroducirse posteriormente. 
Existen pocas recomendaciones sobre la optimización de la medicación después de CB. La reducción del tamaño gástrico, la ausencia de un pH ácido y/o la malabsorción, pueden alterar la absorción de fármacos, disminuyendo su eficacia o aumentando el riesgo de efectos adversos. La disminución de la superficie de absorción afecta sobre todo a los preparados de liberación prolongada y a las formulaciones entéricas, por lo que se recomienda usar formas de liberación rápida, aumentando la frecuencia de administración. Con respecto a los fármacos absorbidos en el estómago o en el duodeno, pueden valorarse rutas alternativas de administración ${ }^{43}$. Se deben evitar la aspirina y el resto de antiinflamatorios no esteroideos por su potencial ulcerógeno, siendo recomendable la administración de protectores gástricos en los primeros meses postoperatorios. En caso de estreñimiento, se aconseja no utilizar laxantes osmóticos (tipo lactulosa) si se han realizado técnicas derivativas por la posibilidad de inducir un síndrome de dumping. La ingesta de alcohol está especialmente contraindicada, ya que los pacientes con exclusión o resección gástrica presentan, por diferentes mecanismos, un aumento de la toxicidad inducida por etanol ${ }^{3}$ and 23 .

\section{¿Es segura la gestación tras la cirugía bariátrica?}

La pérdida de peso inducida por $\mathrm{CB}$ puede normalizar el hiperandrogenismo, mejorando las irregularidades menstruales y la fertilidad ${ }^{44}$. Hay evidencias de que la gestación tras $\mathrm{CB}$, con un peso estable y un adecuado estado nutricional, es segura y no presenta un mayor índice de DM gestacional respecto a mujeres con normopeso de la misma edad ${ }^{45}$. Por otra parte, las mujeres obesas tratadas con CB tienen menos complicaciones relacionadas con la gestación (DM gestacional, preeclampsia y macrosomía, entre otras) que las obesas no intervenidas ${ }^{46}$.

Las complicaciones potenciales del embarazo tras $\mathrm{CB}$ incluyen vómitos persistentes, sangrado gastrointestinal, anemia, retraso del crecimiento intrauterino, deficiencias de micronutrientes y defectos del tubo neural, por lo que debe considerarse un embarazo de alto riesgo. Es imprescindible una estrecha supervisión clínica con controles analíticos trimestrales para adaptar el plan de alimentación y de suplementación a los requerimientos individuales de cada paciente ${ }^{46}$ (tabla 7).

Tabla 7. Gestación y cirugía bariátrica

Recomendaciones

Retrasar la gestación hasta 18 o 24 meses después de la cirugía

Evitar la gestación en periodos de pérdida de peso

Asegurar un estado nutricional óptimo, especialmente en las pacientes con antecedentes de cirugía derivativa

Realizar un control rutinario del peso y un control analítico trimestral exhaustivo durante la gestación

Administrar suplementos preconcepcionales de ácido fólico

Administrar suplementos gestacionales de ácido fólico, yodo, hierro, vitamina $\mathrm{B}_{12}$, calcio y proteínas, incluso a dosis mayores de las habituales debido al mayor volumen de distribución

Evitar dosis elevadas de vitamina $\mathrm{A}$ y $25-\mathrm{OH}-\mathrm{D}_{3}$ por su potencial efecto teratógeno

Evitar el test de sobrecarga oral de glucosa para el cribado y el diagnóstico de DM gestacional (puede determinar hipoglucemias reactivas intensas)

\section{Conflicto de intereses}

Los autores declaran no tener ningún conflicto de intereses.

\section{Anexo 1.}

Juan José Arrizabalaga, Assumpta Caixàs, Alfonso Calañas, Fernando Cordido, Guillem Cuatrecasas, María Jesús Díaz-Fernández, Pedro Pablo Garcia Luna, Albert Goday, Albert Lecube, Lluis Masmiquel, Susana Monereo, María José Morales, José Moreiro, Basilio Moreno, Wifredo Ricart, Josep Vidal. 
1. J. Salas-Salvadó, M.A. Rubio, M. Barbany, Moreno B, Grupo colaborativo de la SEEDO. Consenso SEEDO 2007 para la evaluación del sobrepeso y la obesidad y el establecimiento de criterios de intervención terapéutica. Med Clin (Barc), 128 (2007), pp. 184-196.

2. J.J. Arrizabalaga, L. Masmiquel, J. Vidal, A. Calañas-Continente, M.J. Díaz-Fernández, P.P. García-Luna, et al. . Recomendaciones y algoritmo de tratamiento del sobrepeso y la obesidad en personas adultas. Med Clin (Barc), 122 (2004), pp. 104-110.

3. J.I. Mechanick, R.F. Kushner, H.J. Sugerman, J.M. Gonzalez-Campoy, M.L. Collazo-Clavell, S. Guven, American Association of Clinical Endocrinologists, et al. The Obesity Society, and American Society for Metabolic \& Bariatric Surgery medical guidelines for clinical practice for the perioperative nutritional, metabolic, and nonsurgical support of the bariatric surgery patient. Endocr Pract, 14 (Suppl 1) (2008), pp. 1-83.

4. E.J. DeMaria. Bariatric surgery for morbid obesity. N Engl J Med, 356 (2007), pp. 2176-2183.

5. L. Sjöström, A.K. Lindroos, M. Peltonen, J. Torgerson, C. Bouchard, B. Carlsson, et al. Lifestyle, diabetes and cardiovascular risk factors 10 years after bariatric surgery. N Engl J Med, 351 (2004), pp. 2683-2693.

6. J.B. Dixon, P.E. O'Brien, J. Playfair. Adjustable gastric banding and conventional therapy for type 2 diabetes: a randomized controlled trial. JAMA, 299 (2008), pp. 316-323.

7. H. Buchwald, Y. Avidor, E. Braunwald, M.D. Jensen, W. Pories, K. Fahrbach, et al. Bariatric surgery: a systematic review and meta-analysis. JAMA, 292 (2004), pp. 1724-1737.

8. J.L. Colquitt, J. Picot, E. Loveman, A.J. Clegg. Surgery for obesity. Cochrane Database Syst Rev (2009), p. CD003641.

9. L. Sjöström, K. Narbro, C.D. Sjöström, K. Karason, B. Larsson, H. Wedel, et al. Effects of bariatric surgery on mortality in Swedish obese subjects. N Engl J Med, 357 (2007), pp. 741-752.

10. T.D. Adams, R.E. Gress, S.C. Smith, R.C. Halverson, S.C. Simper, W.D. Rosamond, et al. Long-term mortality after gastric bypass surgery. N Engl J Med, 357 (2007), pp. 753-761.

11. A. Calañas-Continente, J.J. Arrizabalaga, A. Caixàs, F. Cordido, Grupo de trabajo sobre Obesidad de la Sociedad Española de Endocrinología y Nutrición. Recomendaciones diagnósticas y terapéuticas en el sobrepeso y la obesidad durante la adolescencia. Med Clin (Barc), 135 (2010), pp. 265-273.

12. American Diabetes Association. Standars of medical care in diabetes-2009. Diabetes Care, 32 (Suppl 1) (2009), pp. S13-S61.

13. H. Buchwald, R. Estok, K. Fahrbach, D. Banel, M.D. Jensen, W.J. Pories, et al. Weight and type 2 diabetes after bariatric surgery: Systematic review and meta analysis. Am J Med, 122 (2009), pp. 248-256.

14. S. Sánchez Zaldívar, F. Arias Horcajadas, J.J. Gorgojo Martínez, S. Sánchez Romero. Evolución de las alteraciones psicopatológicas en pacientes con obesidad mórbida tras cirugía bariátrica. Med Clin (Barc), 133 (2009), pp. 206-212.

15. Sociedad Española de Cirugía de la Obesidad. Recomendaciones de la SECO para la práctica de la cirugía bariátrica (Declaración de Salamanca). Cir Esp, 75 (2004), pp. 312-314.

16. NIH conference. Gastrointestinal surgery for severe obesity. Consensus Development Conference Panel. Ann Intern Med, 115 (1991), pp. 956-961.

17. M.C. Lewis, M.L. Phillips, J.P. Slavotinek, L. Kow, C.H. Thompson, J. Toouli. Change in liver size and fat content after treatment with optifast ${ }^{\circledR}$ very low calorie diet. Obes Surg, 16 (2006), pp. 697-701.

18. J.A. Sallet, J.B. Marchesini, D.S. Paiva, K. Komoto, C.E. Pizani, M.L.B. Ribeiro, et al. Brazilian multicenter study of the intragastric balloon. Obes Surg, 14 (2004), pp. 991-998.

19. B. Ernst, M. Thurnheer, S.M. Schmid, B. Schultes. Evidence for the necessity to systematically assess micronutrient status prior to bariatric surgery. Obes Surg, 19 (2009), pp. 66-73.

20. K. Fujioka. Follow-up of nutritional and metabolic problems after bariatric surgery. Diabetes Care, 28 (2005), pp. 481-484.

21. R.E. Brolin, L.E. Robertson, H.A. Kenler, R.P. Cody. Weight loss and dietary intake after vertical banded gastroplasty and Roux-en Y gastric bypass. Ann Surg, 220 (1994), pp. 782-787.

22. V. Moize, A. Geliebter, M.E. Gluck, E. Yahav, M. Lorence, T. Colarusso, et al. Obese patients have inadequate protein intake related to protein intolerance up to 1 year following Roux-en-Y gastric bypass. Obes Surg, 13 (2003), pp. 23-28.

23. M.A. Rubio, C. Martínez, O. Vidal, A. Larrad, J. Salas-Salvado, J. Pujol, et al. Documento de consenso sobre cirugía bariátrica. SEEDO-SECO. Rev Esp Obes, 4 (2004), pp. 223-249.

24. C.S. Huang, R.A. Forse, B.C. Jacobson, F.A. Farraye. Endoscopic findings and their clinical correlations in patients with symptoms after bypass surgery. Gastrointest Endosc, 58 (2003), pp. 859-866.

25. J. Ahmad, J. Martin, S. Ikramuddin, P. Schauer, A. Slivka. Endoscopic balloon dilation of the gastroenteric anastomotic stricture after laparoscopic gastric bypass. Endoscopy, 35 (2003), pp. 725-728.

26. C.G. Chang, B. Adams-Huet, D.A. Provost. Acute post-gastric reduction surgery (APGARS) neuropathy. Obes Surg, 14 (2004), pp. 182-189.

27. H.J. Sugerman, J.V. Starkey, R. Birkenhauer. A randomized prospective trial of gastric bypass versus vertical banded gastroplasty for morbid obesity and their effects on sweets versus non-sweets eaters. Ann Surg, 205 (1987), pp. 613-624. 
28. G.J. Service, G.B. Thompson, F.J. Service, J.C. Andrews, M.L. Collazo-Clavell, R.V. Lloyd. Hyperinsulinemic hypoglycemia with nesidioblastosis after gastric bypass surgery. N Engl J Med, 353 (2005), pp. 249-254.

29. H.J. Sugerman, W.H. Brewer, M.L. Shiffman, R.E. Brolin, M.A. Fobi, J.H. Linner, et al. A multicenter, placebocontrolled, randomized, double-blind, prospective trial of prophylactic ursodiol for the prevention of gallstone formation following gastric-bypass-induced rapid weight loss. Am J Surg, 169 (1995), pp. 91-96.

30. R. Kushner. Managing the obese patient after bariatric surgery: a case report of severe malnutrition and review of the literature. JPEN J Parenter Enteral Nutr, 24 (2000), pp. 126-132.

31. R.D. Bloomberg, A. Fleishman, J.E. Nalle, D.M. Herron, S. Kini. Nutritional deficiencies following bariatric surgery: what have we learned? Obes Surg, 15 (2005), pp. 145-154.

32. J. Ocón Bretón, S. Pérez Naranjo, S. Gimeno Laborda, P. Benito Ruesca, R. García Hernández. Efectividad y complicaciones de la cirugía bariátrica en el tratamiento de la obesidad mórbida. Nutr Hosp, 20 (2005), pp. 409414.

33. M. Muñoz, F. Botella-Romero, S. Gómez-Ramírez, A. Campos, J.A. García-Erce. Iron deficiency and anaemia in bariatric surgical patients: causes, diagnosis and proper management. Nutr Hosp, 24 (2009), pp. 640-654.

34. B.M. Rhode, P. Arseneau, B.A. Cooper, M. Katz, B.M. Gilfix, L.D. MacLean. Vitamin B12 deficiency after gastric surgery for obesity. Am J Clin Nutr, 63 (1996), pp. 103-109.

35. G.H. Slater, C.J. Ren, N. Siegel, T. Williams, D. Barr, B. Wolfe, et al. Serum fat-soluble vitamin deficiency and abnormal calcium metabolism after malabsorptive bariatric surgery. J Gastrointest Surg, 8 (2004), pp. 48-55.

36. V. Ruiz-Esquide, P. Peris, L. Gifre, G.N. Guañabens. Alteraciones del metabolismo óseo en la cirugía bariátrica. Med Clin (Barc), 136 (2011), pp. 215-221.

37. L. Newbury, K. Dolan, M. Hatzifotis, N. Low, G. Fielding. Calcium and vitamin D depletion and elevated parathyroid hormone following biliopancreatic diversion. Obes Surg, 13 (2003), pp. 893-895.

38. N. Scopinaro, G.F. Adami, G.M. Marinari, E. Gianetta, E. Traverso, D. Friedman, et al. Biliopancreatic diversion. World J Surg, 22 (1998), pp. 936-946.

39. P. Marceau, F.S. Hould, S. Simard, S. Lebel, R.A. Bourque, M. Potvin, et al. Biliopancreatic diversion with duodenal switch. World J Surg, 22 (1998), pp. 947-954.

40. M.A. Bock. Roux-en-Y gastric bypass: the dietitian's and patient's perspectives. Nutr Clin Pract, 18 (2003), pp. 136-140.

41. Lehman center weight loss surgery expert panel. Commonwealth of Massachusetts Betsy Lehman Center for patient safety and medical error reduction. Expert panel on weight loss surgery executive report. Obes Res, 13 (2005), pp. 205-226.

42. M.J. Morales, M.J. Díaz-Fernández, A. Caixàs, A. Goday, J. Moreiro, J.J. Arrizabalaga, et al. Tratamiento quirúrgico de la obesidad: recomendaciones prácticas basadas en la evidencia. Endocrinol Nutr, 55 (Suppl 3) (2008), pp. 1-25.

43. A.D. Miller, K.M. Smith. Medication and nutrient administration considerations after bariatric surgery. Am J Health Syst Pharm, 63 (2006), pp. 1852-1857.

44. H. Escobar-Morreale, J.I. Botella-Carretero, F. Alvarez-Blasco, J. Sancho, J.L. San Millán. The polycystic ovary syndrome associated with morbid obesity may resolve after weight loss induced by bariatric surgery. J Clin Endocrinol Metab, 90 (2005), pp. 6364-6369.

45. E. Sheiner, J.S. Abramowicz, I. Levy, M. Katz, M. Mazor. Pregnancy outcome of patients with gestational diabetes mellitus following bariatric surgery. Am J Obstet Gynecol, 194 (2006), pp. 431-435.

46. I. Guelinckx, R. Devlieger, G. Vansant. Reproductive outcome after bariatric surgery: a critical review. Hum Reprod Update, 15 (2009), pp. 189-201. 